\title{
Optical design teaching by computing graphic methods
}

D. Vazquez-Molini, J. Muñoz-Luna, A. FernandezBalbuena, A. Garcia-Botella, P. Belloni, et al.

D. Vazquez-Molini, J. Muñoz-Luna, A. A. Fernandez-Balbuena, A. GarciaBotella, P. Belloni, J Alda, "Optical design teaching by computing graphic methods," Proc. SPIE 8481, Optics Education and Outreach II, 84810W (15 October 2012); doi: 10.1117/12.932266

SPIE Event: SPIE Optical Engineering + Applications, 2012, San Diego, California, United States 


\title{
Optical design teaching by computing graphic methods
}

\author{
D. Vazquez-Molini*a, J. Muñoz-Luna ${ }^{\mathrm{a}}$, A. A. Fernandez-Balbuena ${ }^{\mathrm{a}}$, \\ A. García-Botella ${ }^{\mathrm{b}}, \mathrm{P}$. Belloni ${ }^{\mathrm{c}}, \mathrm{J}^{\mathrm{A}}$. Alda ${ }^{\mathrm{a}}$ \\ ${ }^{a}$ University Complutense of Madrid, School of Optics, Dept. of Optics, Madrid, Spain \\ b Dpto. Física Aplicada Recursos Naturales, Univ. Politécnica de Madrid, E.T.S.I. de Montes, \\ Ciudad Universitaria. 28040 Madrid, Spain \\ ${ }^{\mathrm{c}}$ Fakultät Witschaftsingenieurwesen, Hochschule Furtwangen, \\ 78120 Furtwange. Germany. \\ *dvazquez@fis.ucm.es; Phone+34 913946890; \\ http://portal.ucm.es/web/iluminacionycolor
}

\begin{abstract}
One of the key challenges in the teaching of Optics is that students need to know not only the math of the optical design, but also, and more important, to grasp and understand the optics in a three-dimensional space. Having a clear image of the problem to solve is the first step in order to begin to solve that problem. Therefore to achieve that the students not only must know the equation of refraction law but they have also to understand how the main parameters of this law are interacting among them. This should be a major goal in the teaching course. Optical graphic methods are a valuable tool in this way since they have the advantage of visual information and the accuracy of a computer calculation.
\end{abstract}

Keywords: ray-tracing, optical design, refraction, reflection, Snell law.

\section{INTRODUCTION}

For science and technology students, understanding what a mathematical equation means is not always an easy task. Also for whose teaching these subjects, the communication of the importance of a given equation and the relation of their constituents is also a challenging job. In optics this effort is even more important. Specially, when students try to understand how an optical system is working. Fortunately, optics, by nature, is typically linked with a visual representation of the light propagation and its interaction with nature. This fact makes explanations close to everyday phenomena. Then, light propagation is easily explained, within the geometrical optics framework, as trajectories that we usually name as rays. These rays become straight lines when light propagates through uniform and homogeneous media. Even more, when explaining the basics of optical design, graphical schemes and plots are relevant tools to properly analyse the situation. In the past, around 50 years ago, before computing tools were extensive and essential in scientific and industrial environments, graphic methods were used very often [1-4] when equations and numeric calculus became complex. At that time, graphical methods had advantages over numerical ones: They were fast and easy to use, and its visual information was a very good way to get information [5-12]. When a student was working on a specific problem he/she could visualise the problem graphically, helping him/her to solve the equations. However, in those years graphic methods had an important inconvenience related with the lack of accuracy. When computers became cheaper and faster they spread its presence in all scientific and technological offices and classical graphic methods were forgotten. Graphic ability of that first computers was very much reduced and software interface was quite unfriendly. Nowadays computer developments have made possible working with software with an intuitive user friendly interface. Besides graphic capabilities have improved greatly. Optical design [7-9] and other fields [10] have benefited from such a leap in performance, enabling the sophisticated tools of computer-aided design that we know nowadays. Besides, graphical human interfaces have been developed to offer to the engineer and designer supplementary tools to interact with the results of the analysis in a very intuitive way. In this contribution we describe some simple solutions to the ray tracing problem that are solved using graphic methods. We show how reflection, refraction, and the characterization of optical surfaces can be addressed quite efficiently using graphics methods. 


\section{COMPUTER GRAPHICAL METHODS IN OPTICS}

When a student deals for the first time with the Snell Law he understands very fast how to work with it analytically, but usually he/she does not really understand what is behind the numbers and how the phenomena is working. For instance, it is not clear from the mathematical expression what happens when incident angles is small, or what happens in the case of incidence angles above the critical angle. In this paper we describe all the cases that we can find in a simple ray tracing process. The problems that we will evaluate graphically are:

- Evaluation of the reflected ray for a plane mirror

- Evaluation of the refracted ray for a plane interface

- Evaluation of the normal to the surface at an arbitrary point of incidence.

\subsection{Reflected ray calculation}

The reflection of light on a polished surface is a very simple problem both mathematically and graphically. In history we can find a lot of problems solved by this graphic method [11-12]. Graphically, to find the location and direction of any reflected ray we only need to apply an operation of symmetry, where the axis of symmetry is the normal to surface. In this case we apply this symmetry operation on a plane defined by the vector normal to the surface and the incident ray. However, if we think of a plane mirror as an image-forming system, then the surface acts as a plane of symmetry for the transformation of the object into an image. Even more, if the surface is used as a symmetry axis the obtained symmetric ray can still be used to obtain the reflected ray. To do that it will be necessary to extend the ray to the hemisphere where it is belong. This operation is integrated in all software CAD package as a function usually named as "mirror" operation. With this method it will be very easy for students to do a ray-tracing, for example, in a compound parabolic concentrator or an ellipsoidal system with a mirror treatment.

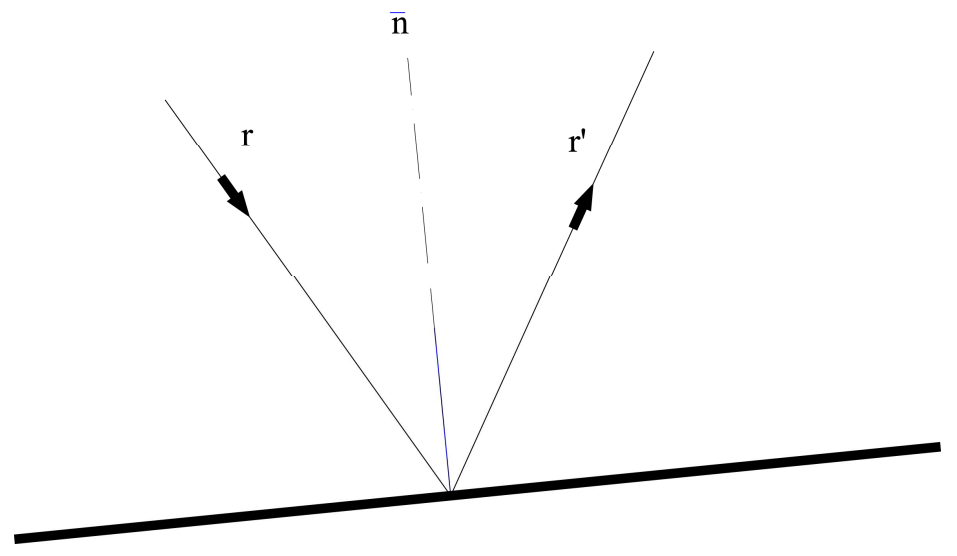

Figure 1: Reflected ray calculation by using a symmetry operation.

As an example, we show in figure 2 the ray-tracing obtained for a compound parabolic concentrator (CPC) using the graphic method showed previously. A CPC consist on a tilted parabola with foci at the exit of the system. This optical component is designed to accept light entering within the preset angle of acceptance. In Fig. 2 this angle of acceptance has a value of $30^{\circ}$. If light is entering from the optical axis till $30^{\circ}$ is all accepted. If the angle is higher than $30^{\circ}$ is easy to demonstrate that the ray is rejected. For this reason a CPC has a $2 \mathrm{D}$ efficiency of $100 \%$. 


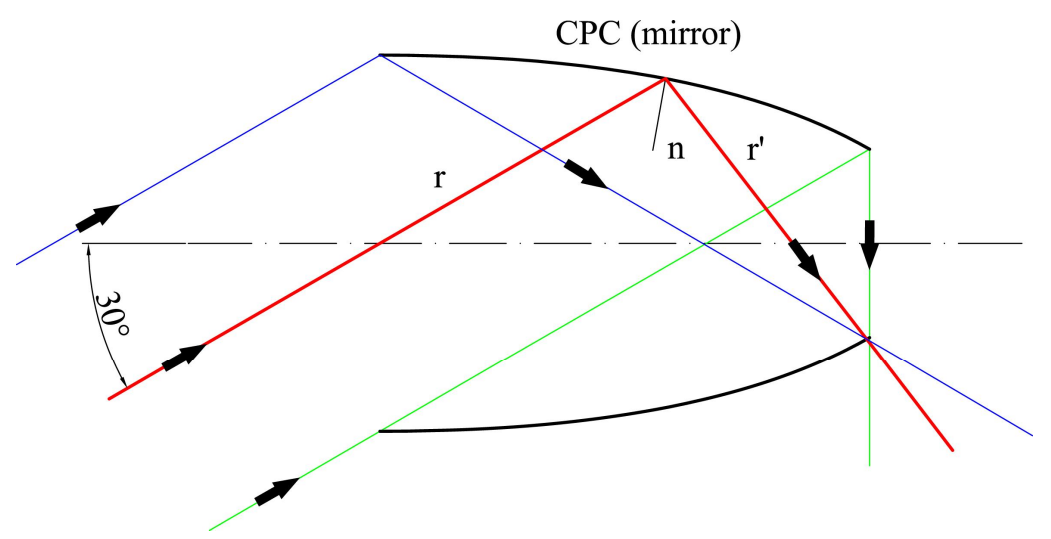

Figure 2: Reflected ray calculation on a compound parabolic concentrator.

\subsection{Refracted ray calculation}

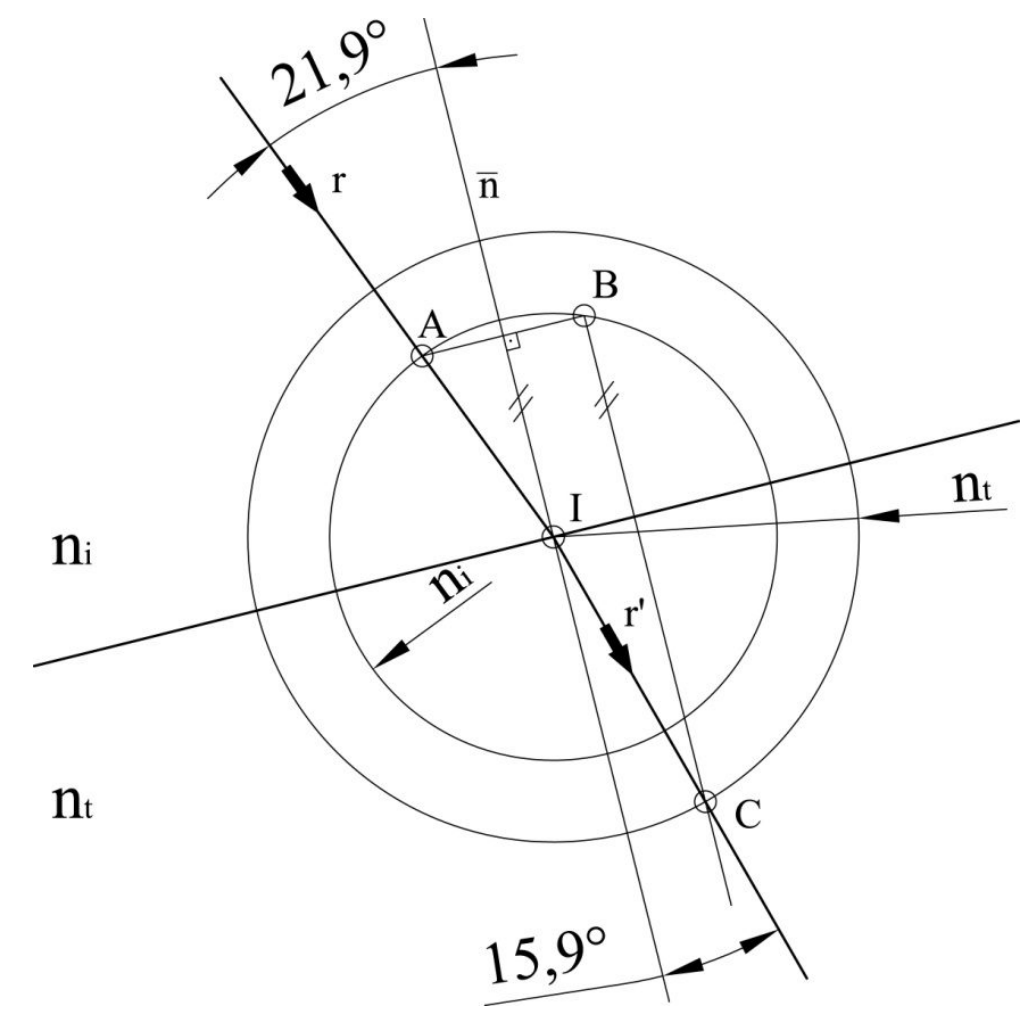

Figure 3: Refracted ray calculation by using a simple graphical method.

The case of refraction involves the graphical resolution of the Snell law. In this case, we have two materials with index of refraction $n_{i}$ and $n_{t}$ are given. First of all we define the point of incident on the surface separating these two materials. This point is labelled as I in figure 3. Now we draw two concentric circles being I the centre of these two circles. The radii of each circle are the values of the index of refraction of the two media involved in the refraction. We define point $\mathrm{A}$ as the intersection of the inciding ray with the circle having a radius equal the index of refraction of the first medium, $n_{i}$. Then, we draw a segment, $A B$, perpendicular to the normal to the surface and ending at the same circle. The next step 
is to draw another line, now parallel to the normal to the surface, beginning at point $\mathrm{B}$ and ending at a point $\mathrm{C}$ that belongs to the circle having a radius equal to the index of refraction of the second medium. Finally, the ray after refraction passes through point C, and it is therefore defined by the segment IC. Students know that Snell law is given as,

$$
n_{i} \sin \left(\varepsilon_{1}\right)=n_{t} \sin \left(\varepsilon_{2}\right),
$$

From a graphical standpoint and, by analyzing figure 3, we can see that the distance from point A to the normal to the surface is equal to the left hand side of Snell law. Analogously, the distance between point $\mathrm{C}$ and the normal to the surface is equal to right side hand of equation (1). Finally, by graphical construction we may see that these two previous distances are equal.

As an example, we show in figure 4 a simple case where a ray path changes for two different wavelengths. Two rays fall in a spherical lens made with polycarbonate with a refraction index in the visible range of 1.63 at $380 \mathrm{~nm}$ and 1.57 when the wavelength is $780 \mathrm{~nm}$. In this example we only show the refraction graphic method for the first refraction in the first surface of the lens but the graphic method is applied in each interface. The chromatic dependence is graphically represented by the two outer circles that appear at the incidence point. Each one of these outer circles has an index of refraction different, corresponding with the two wavelengths of interest. When reaching the second surface the graphical construction method has to be separately applied to each one of the intersection points obtained from the two rays generated at the first surface. Students must be careful since in the second surface of the lens the ray pass from a high refraction index to another lower refraction index. When students have done all refractions for the rays they can easily check how the chromatic aberration affects to the raytracing.

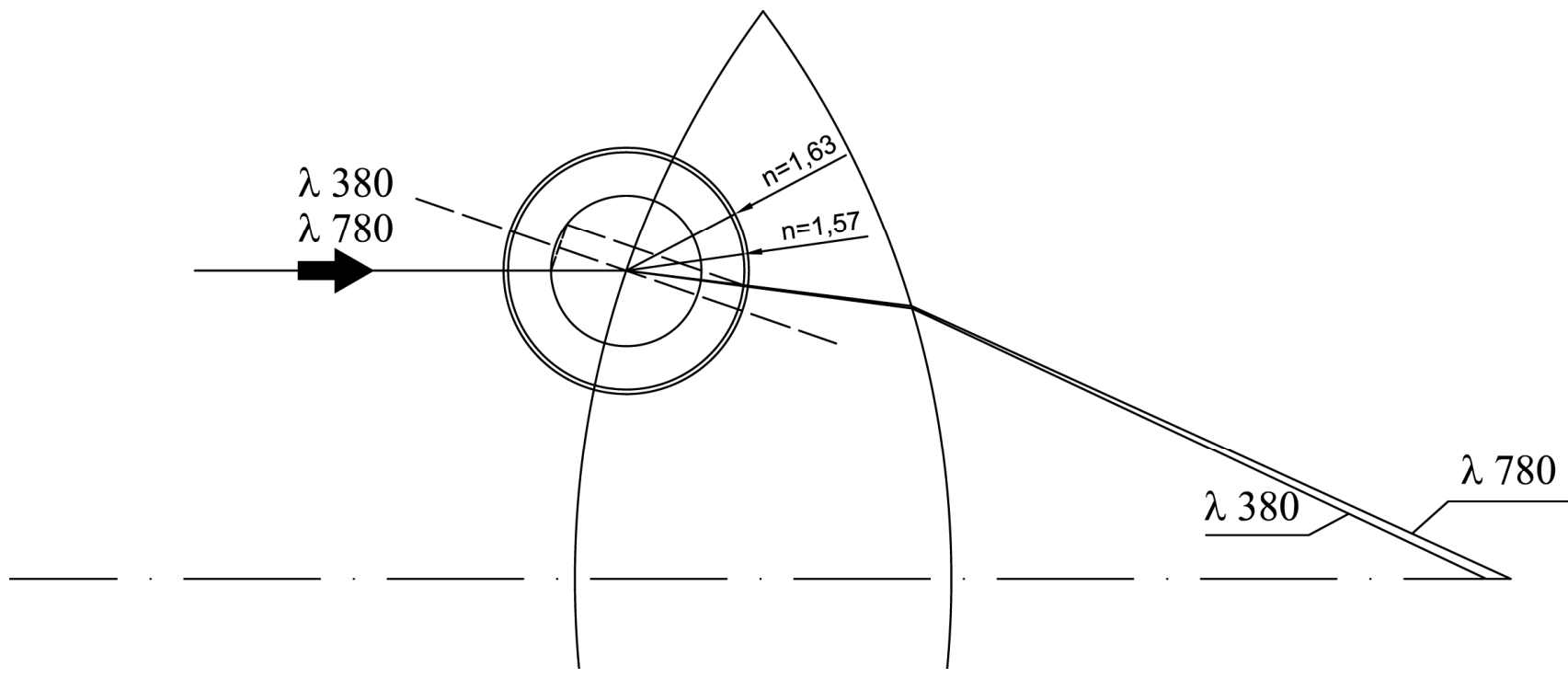

Figure 4: Refracted ray calculation for two wavelengths in a lens

Limit angle condition appears when light travels from an index to another lower index of refraction. If the angle of incidence is larger than the limit angle, the ray will be reflected and not refracted as showed in figure 5 . This situation is depicted by the students when, in lens analyzed previously they find a ray in the second surface of the lens inciding at a large angle. If the angle is larger than the limit angle, it is not possible to draw a line parallel to the normal to the surface that intersect the circle having a radius equal to the index of the second medium. It is also possible to conclude that limit angle condition only happen when the index of refraction of the second medium is smaller than the index of refraction of the first medium. 


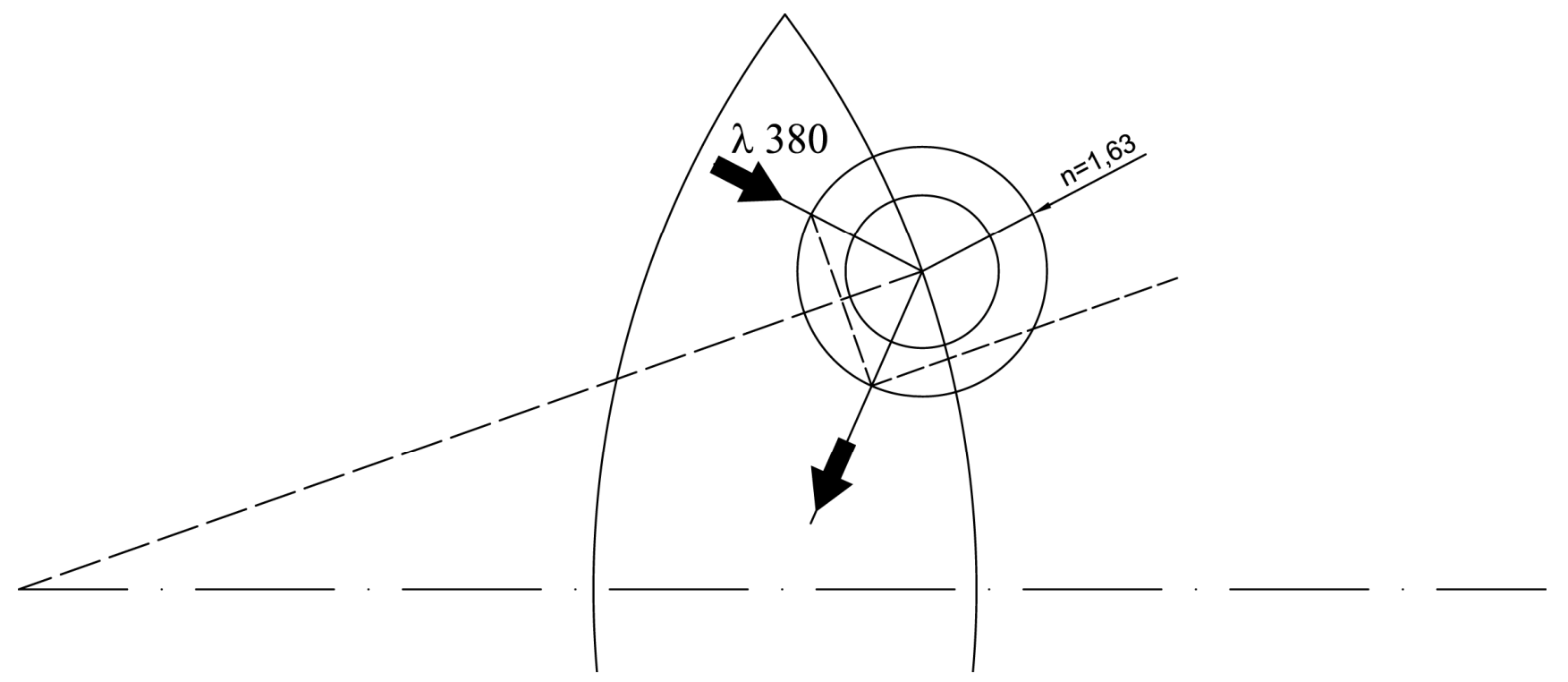

Figure 5: Total internal reflection in a second surface of a lens.

\subsection{Calculation of the optical surface}

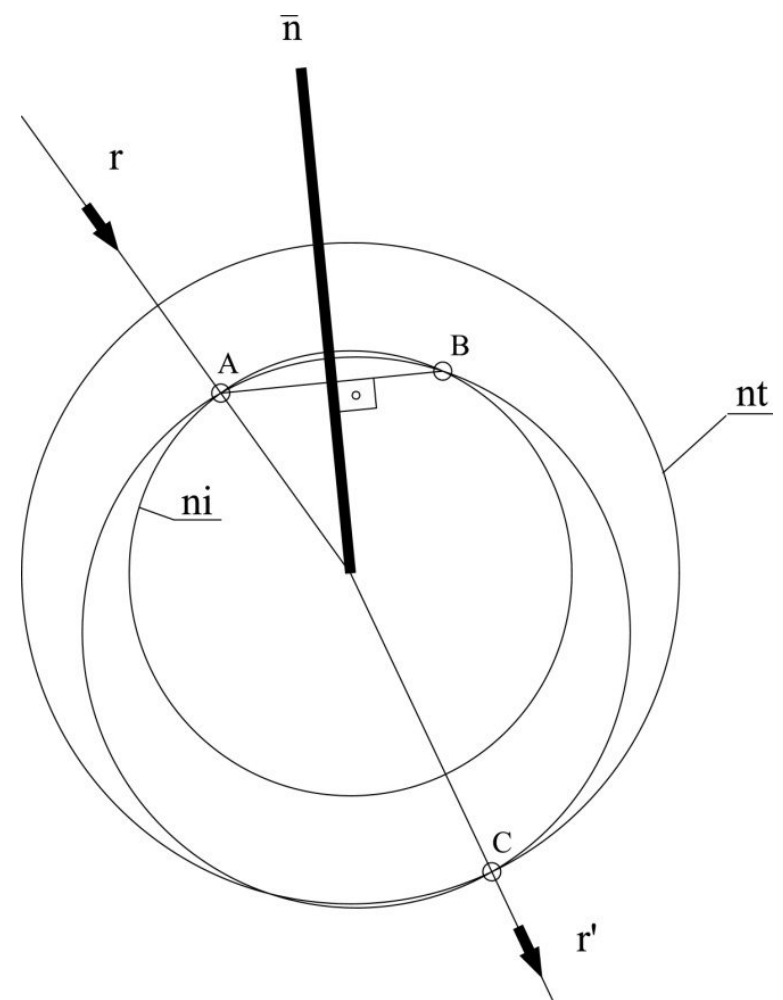

Figure 6: Graphic method for calculating the surface normal at incident point.

Sometimes, optical design problems begin with the specification of the final location of the refracted, or reflected, light refracted by a given and still unknown surface. Then, the surface needs to be found. Graphic methods may help to perform this task very easily. When we know the point where light is inciding on an optical surface, and the direction of 
the incident and refracted rays are also known, we typically need to find out how would be the surface in that point, i.e. what will be the normal of this surface. In this case, from a classical point of view, students should define a geometry or even use a software program to define it. This can be a quite challenging task if addressed analytically. It will spend a lot or time and effort. However, a graphic method, as the one presented in this paper can do the job quite quickly and in a very straightforward way. Now we have the incoming direction labelled as $r$ in figure 6 , and an outgoing direction. We also have a point of incidence. This point of incidence is the intersection between rays $r$ and $r$ '. Then, from figure 3 we have seen that $\mathrm{ABC}$ is a right angle. At the same time $\mathrm{A}$ and $\mathrm{B}$ belong to the circle having a radius equal to the index of refraction of the first medium, $n_{i}$, meanwhile $\mathrm{C}$ lies on a concentric circle having a radius equal to the index of refraction of the second medium, $n_{t}$. However, we still know only the location of $\mathrm{A}$ and $\mathrm{C}$. To know it we can use a circle having as diameter the segment AC. This circle contains all the right triangles with $\mathrm{AC}$ as hypotenuse. At the same time this circle will intersect the circle having a radius equal to $n_{i}$. The intersection will be a two points: A and B. Therefore, we already know the location of $\mathrm{A}, \mathrm{B}$, and $\mathrm{C}$. As far as the normal to surface has to be parallel to $\mathrm{BC}$ and perpendicular to $\mathrm{AB}$ and passing through the intersection between $r$ and r', we can say that the problem has been already solved graphically.

Now again, we show an example of how to calculate a plane-convex lens free of spherical aberration. To simplify the situation we assume that the first surface is a plane diopter and that incidence is represented by a collection of parallel rays coming from infinity. In this situation it is easy to find the right second surface by means of the graphic method described before. The starting point is located at the optical axis because in such a way it is possible to define the thickness of the lens. Step by step, the incidence is displaced away from the center in order to find the directions of the normal to the surface. We can see that the method is sequential, so after the first segment you can find the second one and successively until the edge of the lens is obtained. Figure 7 shows the final result of the graphical calculation.

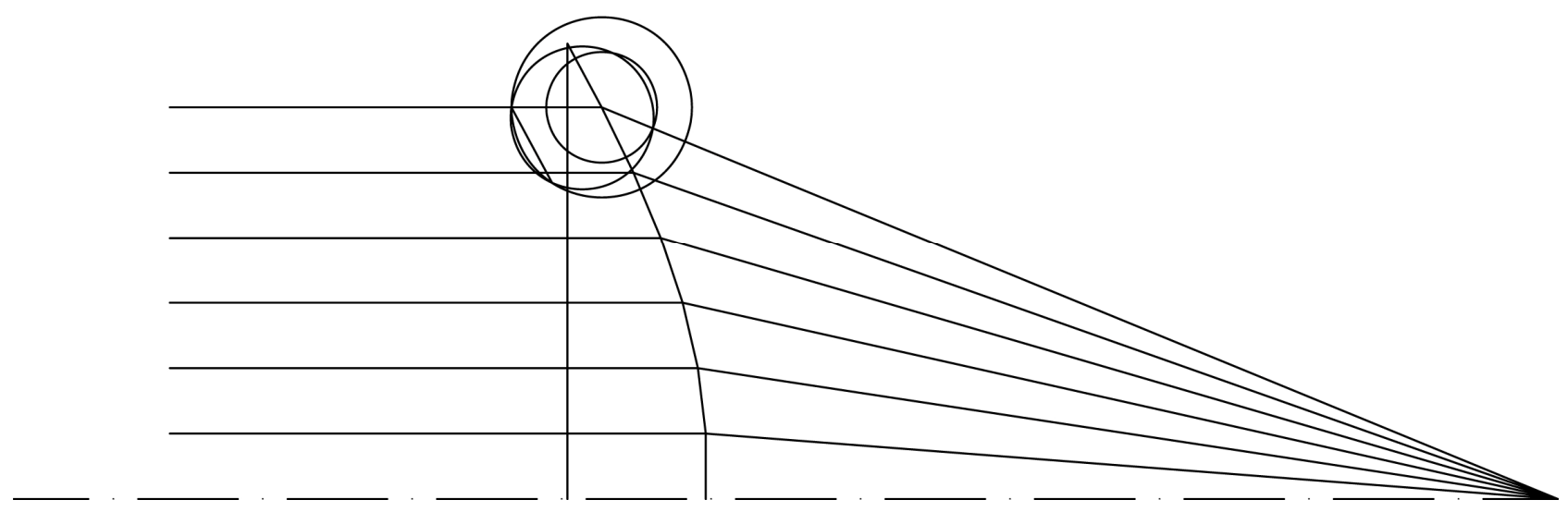

Figure 7: Graphic method for designing the second surface of a plano-conex lens without spherical aberration.

\section{CONCLUSIONS}

This paper reviews the relationships between teaching CAD and optics. We revisited the basics laws of optics from a different perspective, and we propose to use simple graphical methods based on symmetry properties when working with $\mathrm{CAD}$ systems in order to propagate light through optical optical systems. Also we show some interesting examples of useful optical systems that can be easily designed or evaluated with a graphical method. We believe that if students can work with CAD systems, which are very intuitive and produce results with high accuracy, they will understand better how optics works and how it is possible to analyze graphically the raytracing through refracting or reflecting optical surfaces. These methods can be of great help when introducing optics to experienced mechanical designers, or any other professional used to the graphical tools of modern CAD packages. 


\section{ACKNOWLEDGEMENTS}

This contribution has been supported by Science and Innovation Ministry of Spain with the projects HAR2009-12862 and ENE2009-14340.

\section{REFERENCES}

[1]

[2]

[3]

$[4]$

R. E. Lewis, "Graphical ray-Trace and Surface Generation Methods for Aspheric Surfaces", J. Opt. Soc. Am. 41, 456-459,(1951).

[6] H. V. Shelling, "A Simple Graphic Metohd for the Additive Mixture of Two Colors on the CIE Chromaticity Diagram,” J. Opt. Soc. Am., 43, 706-707 (1953)

[7] Encyclopaedia Britannica "Ray-Tracing Methods", http://www.britannica.com/EBchecked/topic/430511/optics/37949/Ray-tracing-methods, accesed on-line ( $9^{\text {th }}$ July, 2012)

[8] Alda, J., Arasa, J., "Paraxial ray-tracing," in Encyclopedia of Optical Engineering, Marcel-Dekker, (2004)

[9] Arasa, J. Alda, J., "Real ray-tracing," in Encyclopedia of Optical Engineering, Marcel-Dekker, (2004)

[10] van Monckhoven D., "Photographic Optics," Ed R. Hardwicke (1867)

[11] Doyle, W. T. "Graphical approach to Fresnel's equations for reflection and refraction of light," Am. J. Phys., 48, 643-647 (1980).

[12] Block, A., "Graphical construction for refracted ray," J. Sci. Instrum. 13, 302-303, (1936) 